\title{
'X' marks the spot: Transferring dig site coordinates from maps to Google Earth
}

Nicole Jaremco, Manisha Saraswat, Howard Gibbins, Philip J. Currie, Clive Coy

${ }^{1}$ Department Biological Sciences, University of Alberta

\begin{abstract}
Dinosaur Provincial Park has been a popular site for palaeontological digs for many years. Over time, the many quarries and bone beds uncovered have had their locations marked on large paper topography maps. Unfortunately, many dig sites have been lost due to poor documentation. Some sites have been abandoned for years. The high erosion levels of the park ( $2-4 \mathrm{~mm}$ yearly) continually both destroys dig sites and uncovers new fossils. To help recover old, unused dig sites, the coordinates of the sites marked on the old paper maps were uploaded to Google Earth Pro for easy access. Unfortunately, the points had to first be transferred to clear mylar maps, because the original paper maps lacked longitude and latitude measurements. This was accomplished by matching the topography when the scale of the maps differed, and by overlaying the clear maps on the paper maps when they did not. The distance of each point from a line of longitude or latitude was found using a ruler (each $\mathrm{mm}$ measured on the map representing $10 \mathrm{~m}$ in the park) and used to calculate their coordinates. After the coordinates were found, they were recorded in a Google SpreadSheet. Once this was completed for all 462 points, they were uploaded to Google Earth Pro. The purpose of this project was to provide more easily accessible records of dig sites and prevent further record loss as the old paper maps age and their condition deteriorates. The massive paper maps are unwieldy and impractical to use in the field, and something more compact is needed. Google Earth is easily accessed on a computer or cell phone, and the points will not be lost due to physical damage, degradation, or misplacement of the records. In addition, it takes up far less space in digital form, and thus is better for field work than the original maps.
\end{abstract}

Key words:

Dig sites, points, coordinates, maps, Google Earth, transfer, topography

Cite as: Jaremco, N., Saraswat M., Gibbins, H., Currie, P.J., Coy, C. 2019. X' Marks the Spot: Transferring Dig Site Coordinates from Maps to Google Earth. Alberta Academic Review, Vol 2 (2) 45-46, WISEST Special Issue (non peer-reviewed), DOI 10.29173/aar58. 


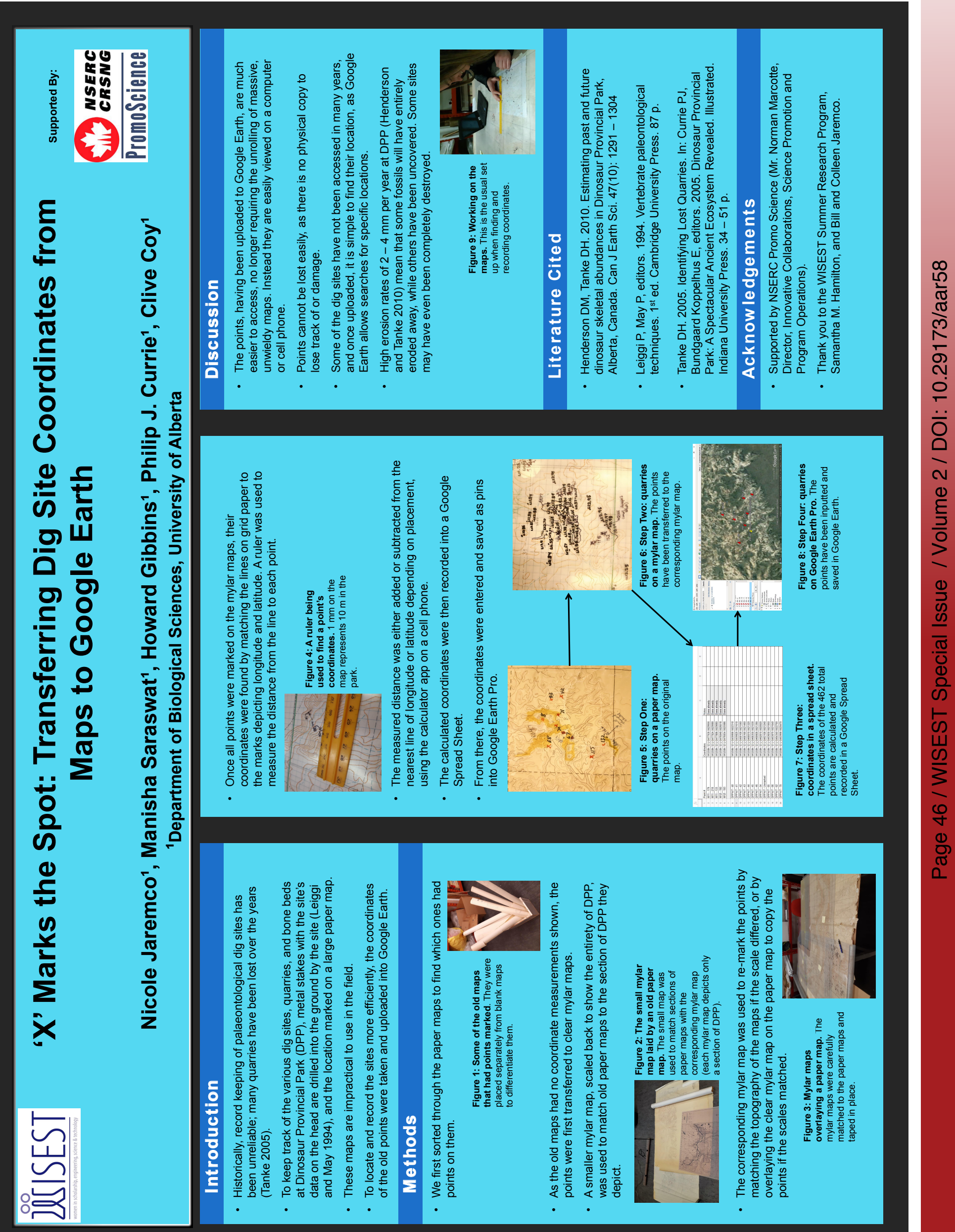

